Oskar Reichmann

\title{
DER FRÜHNEUHOCHDEUTSCHE WORTSCHATZ AUS KULTURGESCHICHTLICHER SICHT
}

\section{Vorbemerkungen}

Meine gesamte lexikographische Tätigkeit ${ }^{1}$ ist von folgenden Basisannahmen über die Lexik von Sprachen vom Typ der europäischen her motiviert: Alles Relevante, was die Natur dem Menschen gleichsam vorgängig entgegenstellt (was auch immer dies sein $\mathrm{mag}^{2}$ ), und alles Relevante, was Menschen an Kultur, also z. B. an Sachgütern, an gesellschaftlichen Einrichtungen, an Erkenntnissen, Ideologien und sozialen Handlungstypen hervorbringen, findet seinen Niederschlag in Einzelsprachen, speziell in deren Lexikon. "Seinen Niederschlag finden“ heißt: Es wird in der gesellschaftlichen Kommunikation konstituiert, in lexikalischen Zeichen gefasst, in U mlauf gehalten und durch den Gebrauch zur mehr oder weniger festen sozialen N orm erhoben. Es wird außerdem - und das ist mindestens ebenso wichtig - immer in einer bestimmten Weise, nämlich aus der jeweiligen kulturellen (das ist: aus der zeit-, raum-, schichten-, gruppen-, situationstypischen) Praxis heraus konstituiert und in U mlauf gehalten, hat also einen genuin gesellschaftlichen Status. Mehr noch: Es gibt keine andere, entsprechend leistungsfähige Konstitution von Realität ${ }^{3}$ als die ein-

1 Die hier vorliegende Druckfassung des Tagungsbeitrags behält die Formulierungen der Vortragsfassung großenteils bei.

2 Es ist auf jeden Fall sehr viel weniger, als man gemeinhin denkt; vgl. R. B ar thes 2003, wo es in der "Vorbemerkung" heißt, dass Natur und Geschichte "ständig miteinander verwechselt" würden (S. 7), dann S. 17: „Der Mythos von der conditio humana stützt sich auf eine sehr alte Mystifikation, die seit jeher darin besteht, auf den Grund der Geschichte die Natur zu setzen“.

3 Hier müsste selbstverständlich in z. B. "Realität“ und "R ealitätsbild“, in sog. natürliche und soziale Realität usw. differenziert werden; außerdem wäre im Sinne Wilhelm von $\mathrm{H}$ umbol dts (und der von inm begründeten Tradition) zwischen Sprache im Sinne von ,allgemeinmenschlicher Sprachfähigkeit' als transzendentaler Voraussetzung von Einzelsprache und Einzelsprache unterschieden werden. 
zelsprachliche, innerhalb deren die lexikalische eine herausragende Rolle spielt, und es gibt keine anderen als je bestimmte kulturtypische Weisen solcher Realitätskonstitution. ${ }^{4}$ Einzelsprachlich-lexikalische Gegenstandskonstitute sind damit die primären darstellungs-, erkenntnis-, kommunikations- und symptomfunktionalen Basiseinheiten, in denen sich Kultur abspielt; auf jeden $\mathrm{Fall}$ ist nur in dem Maße, in dem diese Einheiten infolge ihrer Zeichenexistenz überliefert sind und von Philologen wissenschaftlich gesammelt und beschrieben werden, überhaupt etwas über Vergangenheit aussagbar. Dieses Bündel von Basisannahmen ist mein lexkkographisches Manifest. Auch wenn es, etwa von Seiten der Archäologie, der Kunstgeschichte und anderer, weniger oder gar nicht auf sprachliche Überlieferung gestützter Wissenschaften, sowie von Seiten der Philosophie relativiert werden sollte, hat es eine Gültigkeit, die zumindest unter methodischen Aspekten, also hinsichtlich der sprachlichen Überlieferung als Bedingung der Möglichkeit historischer Forschung, nicht bestreitbar sein dürfte.

Der heute weithin akzeptierte Kanon von Fächern, deren Gegenstandszugang in Sprache und über Sprache verläuft, also der Sprach- und Literaturwissenschaft, der Theologie, Philosophie und des Rechtes, aller im engeren und weiteren Sinne geschichtswissenschaftlicher Disziplinen, aller sachgeschichtlicher Fächer (usw.), ist zu wesentlichen Teilen im 19. $\mathrm{J}$ ahrhundert etabliert worden. ${ }^{5}$ Er verhüllt durch seine Gliederungen und durch deren akademisch vermittelte F estigkeit das grundlegende Faktum, dass die Gliederungen historischer Kulturverhältnisse großenteils quer zu heutigen Fachgrenzen liegen. Daraus folgte in der Gründungsphase des Frühneuhochdeutschen Wörterbuches (FWB), dass dies tatsächlich nicht Fachgrenzen des 19. J ahrhunderts übernehmen konnte, sondern ein frühneuhoch deutsches W örterbuch im Sinne von gesamtsprachbezogenem (dazu R eichmann 1990a) Wörterbuch sein musste, also dem Anspruch nach die Überlieferung dieser Sprachstufe in allen ihren Teilepochen, Teilräumen, Textsorten als Basis haben müsse. Der Gegensatz dazu, der also

${ }^{4}$ Diese Betonung des Kulturtypischen richtet sich nicht gegen das hohe Gewicht, das man der menschlichen Sprachfähigkeit (language) zubilligen muss. Sie richtet sich aber gegen einen, wie F. M authner es formuliert, "verstiegenen Kosmopolitismus", da "er nicht zur Sprachgeschichte [führt], weil alle historischen Undinge in ihm begründet sind: Abstammung von Adam und Eva, die ebenso fabelhafte philosophische Grammatik und die Universalsprache der Zukunft (1910/11, S. XV).

5 Für die Germanistik vgl. dazu etwa W. B ahner /W. N eu mann (Hrsg., 1985). 
nicht angestrebt wird, wäre z. B. ein literarisches oder ein auf eine Einzelfachgeschichte bezogenes Wörterbuch, das dann primär nur für Z. B. Literaturwissenschaftler oder Theologen oder Medizinhistoriker brauchbar wäre. Eine derartige Begrenzung hätte den Gegenstand, z. B. semasiologische, onomasiologische, wortbildungsmorphologische Felder, Metaphernund Bildfelder, syntagmatische Formulierungstraditionen usw. nach Gliederungsgesichtspunkten des 19. J ahrhunderts zerschnitten. - Ich komme damit zur Gegenstandsbestimmung des FWB.

\section{Der Gegenstand und die kulturhistorische Perspektive}

Im Folgenden werden die relevantesten Aspekte des Gegenstandes des Wörterbuches nach (eingeklammerten) Zahlen aufgelistet und anschließend in die kulturhistorische Perspektive gestellt.

(1) Das FWB erfasst und beschreibt dem Anspruch seiner Gründer nach alle lexikalischen Einheiten des Frühneuhochdeutschen. Das sind rund 150.000 Lemmazeichen. Bei dieser Zahl ist zu bedenken, dass der Status der einzelnen Einheiten (hinsichtlich Morphologie oder Funktionalität der Wortart, hinsichtlich Einfachheit oder Komplexität der Einheit: Simplex, W ortbildung, Phrasem usw.) unterschiedlich ist; die genannte Zahl ist ferner natürlich durch die Überlieferungsverhältnisse und durch bestimmte lexikographiepragmatische Zwänge bedingt: Nach meinem Urteil sollte man spätestens dann aufhören zu recherchieren, wenn erstens absehbar ist, dass das zutage geförderte Material nicht mehr in einer Gelehrtengeneration beschrieben werden kann, und wenn zweitens die lexikographische Erkenntniskurve ${ }^{6}$ asymptotische Züge annimmt. Die Frage, wie sich die 150.000 Einheiten zu demjenigen verhalten, was "tatsächlich“ vorhanden gewesen ist, bleibt ausgeblendet. - Als Grundlage der Gewinnung des genannten Inventars dient ein Corpus von rund 800 Texten und Textsammlungen von insgesamt etwa 400.000 Seiten U mfang, das alle Teilepochen, alle Räume und alle Textsorten des späten Mittelalters und der beginnenden Neuzeit in corpustheoretisch relativ ausgewogener Weise berücksichtigt und das arbeitstechnisch in bestimmter Weise exzerpiert wurde. $^{7}$

\footnotetext{
6 Zu dieser Kurve s. Reichmann 1990b, S. 1596.

7 Die verschiedenen Modi der Exzerption sind in der "Lexikographischen Einleitung" zu den Bänden 1 (dort speziell S. 43-62) und 3 (dort S. V-XXV) beschrieben, inzwischen nach verschiedenen Dimensionen verfeinert worden.
} 
(2) J ede der 150000 Einheiten wird semasiologisch, also hinsichtlich ihrer Polysemie und aller begründbaren Gebrauchsnuancierungen, beschrieben. Beim durchschnittlichen Ansatz von 2, 3, [... 6 sog. Einzel bedeutungen (= Sememen) kommt man dann logischerweise auf 2, 3, [...], 6 mal 150.000 Bedeutungsansätze, bei Einbezug von Nuancierungen in die Berechnung ergeben sich noch weit höhere, nicht sinnvoll quantifizierbare Zahlen.

(3) Wenn die lexikalische Bedeutungskonstitution, ich hätte auch sagen können, die lexikalisch normierte Bezugnahme auf die reale oder gedachte Welt, immer nur - wie in den Vorbemerkungen gesagt wurde - auf kulturspezifische Weise erfolgt, und wenn das Wörterbuch für alle historischen Disziplinen brauchbar sein soll, dann ist in besonderer Weise auf die soeben angesprochenen semantischen Mehrdeutigkeiten einschließlich ihrer Nuancierungen $z u$ achten, die an die ebenfalls schon angesprochenen Zeit-, Raum-, Sozialgruppen und Sozialsituationen gebunden sind. Der Abstraktionsgrad der Bedeutungsansätze wird infolge dieser, bereits im Programm stehenden semasiologischen Differenzierungen nicht besonders hoch sein können; er darf jedenfalls nicht so hoch sein, dass gerade das Kulturtypische (Geschichtstypische) der Abstraktion zum Opfer fällt. Den Abstraktionsgrad bemessen zu wollen, wie dies (auch in Rezensionen) manchmal als möglich und sinnvoll suggeriert wird, ist infolge der Existenzweise des Gegenstandes, historischer Wortschatz' bereits im Ansatz verfehlt. Aus lexikographiepragmatischen Gründen wird man sogar je nach funktionaler Zugehörigkeit des einzelnen Lemmazeichens, je nach Überlieferung, je nach vermutetem Benutzerinteresse, überhaupt je nach Einzelwort sehr unterschiedlich verfahren müssen und wollen.

(4) Es sind die sozialen Gruppen (so sei hier zusammenfassend für obiges dauernde raum-, zeit-, sozialtypisch usw. gesagt) zu nennen, die als vorwiegende Träger der semantischen Differenzierungen in Betracht kommen, natürlich in ihrer Zeit-, Raum-, Sozialbindung und in ihrer Textsortentypik.

(5) Da keine lexikalische Einheit, auch keine Einzelbedeutung einer lexikalischen Einheit, isoliert von anderen steht, ist jede Einheit pro Bedeutung außer in ihrer semasiologischen auch in ihrer onomasiologischen Vernetzung zu beschreiben: Pro Bedeutung werden also onomasiologische F elder angegeben. 
(6) J ede Einheit wird pro Bedeutung so beschrieben, dass ihre syntaktischen Gebrauchsregeln und die typischen Nutzungen ${ }^{8}$ dieser Regeln erkennbar werden.

(7) J ede der wortgebildeten und noch nicht vollständig demotivierten lexikalischen Einheiten des Frühneuhochdeutschen ist hinsichtlich ihrer wortbildungsmorphologischen Motivation durchschaubar zu machen, da diese nicht ohne Belang für die Bedeutungskonstitution ist (hierzu R eich$\operatorname{mann} 2002 b)$.

(8) All dasjenige, was ich gerade aufgezählt habe, wird so dokumentiert (und zwar im Belegteil der Artikel), dass es jeder kritische Benutzer selbst nachprüfen, im Ernstfall aus dem Belegteil herleiten kann. Dies setzt einen fortwährenden Bezug auf das Corpus und seine Einzeltexte voraus.

Das FWB muss nach dem Vorgetragenen eine Anlage haben, die für jedes der genannten Beschreibungsanliegen mindestens eine (oder mehrere) Informationsposition innerhalb des Artikels aufweist. Im Schema ergibt das dann folgende Grundstruktur der Artikel.

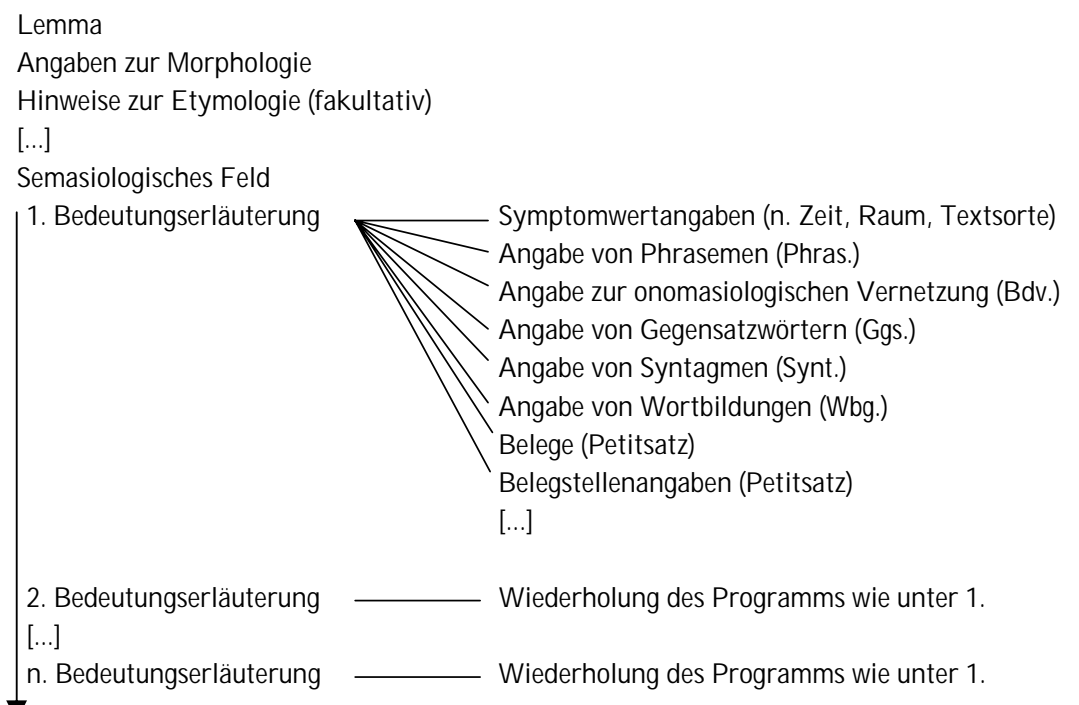

Abb. 1: Schema der Anlage der Artikel des FWB

\footnotetext{
8 Im Hintergrund dieser Formulierung steht die Unterscheidung E. Coser ius in ,System", ,Norm' und ,Parole' (1974), im übrigen ist diese Unterscheidung auch für die meisten anderen Informationspositionen eines Wörterbuchartikels von Bedeutung.
} 
Man erkennt nach der Angabe des Lemmas (das ist genauer: die nach vorgegebenen Regeln aus den Belegschreibungen normalisierte Zeichengestalt) und nach einigen weiteren, hier nicht zu diskutierenden Angaben (zur Morphologie, fakultativ zur Etymologie) die im Vorangehenden unter (1) bis (8) angesprochenen Gegenstandsaspekte als Informationspositionen des Wörterbuches wieder:

- Von oben nach unten findet sich die semasiologische Dimension abgetragen und durch einen Senkrechtpfeil gekennzeichnet. Damit wird auf das Faktum Bezug genommen, dass sprachliche Zeichen systematisch mehrdeutig sind. Der vom Lexikographen angesetzte Grad der M ehrdeutigkeit (Polysemie) spiegelt sich in den Zahlen von 1 bis $n$.

- Dem einzelnen Bedeutungsansatz folgen zunächst die sog. Symptomwertangaben, im FWB realisiert als Angaben zur räumlichen, zeitlichen und textsortenbezüglichen Bindung des Lemmazeichens (jeweils pro Bedeutung).

- Danach stehen die Angaben zur onomasiologischen Vernetzung gemäß dem Faktum, dass ein B egriff systematisch auf verschiedene W eise ausgedrückt werden kann.

- Ihnen folgen die Syntagmen, eng damit verbunden die Angaben zur Wortbildung und schließlich die Belege und die Belegstellenangaben.

Ich habe mich zuletzt mit der Bezugnahme auf Syntagmen, Wortbildungen, Belege (usw.) noch summarischer gefasst als bis dahin, weil ich erstens denke, dass das Schema auch ohne weitere Erklärungen verständlich ist, und weil ich zweitens noch die Veranschaulichung durch ein Beispiel und die Herstellung des Bezuges auf den kulturhistorischen Aspekt schuldig geblieben bin. Als Beispiel dazu möge ein Ausschnitt aus dem Artikel bescheidenheit dienen (Abb. 2).

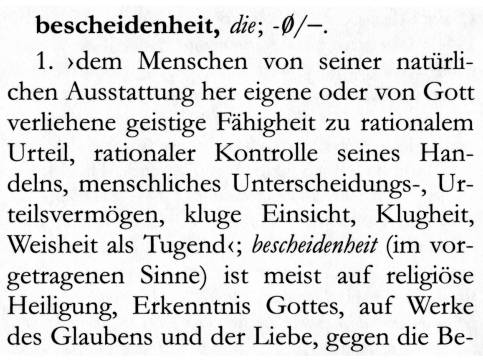
chen Ausstattung her eigene oder von Gott verliehene geistige Fähigheit zu rationalem Urteil, rationaler Kontrolle seines Handelns, menschliches Unterscheidungs-, Urteilsvermögen, kluge Einsicht, Klugheit, Weisheit als Tugend ; bescheidenheit (im vorgetragenen Sinne) ist meist auf religiöse Heiligung, Erkenntnis Gottes, auf Werke des Glaubens und der Liebe, gegen die Be-

gierden gerichtet; seltener bezieht sie sich auf die tägliche Lebenspraxis; schwach belegt sind einige an diese Bedeutungsposition anschließbare Metonymien, z. B. >Unterscheidung«; ,Wissen, Kenntnis von etw.〈; ২Erklärung`; vgl. bescheiden (Adj.) 1; 3 .

- Gehäuft Texte religiösen, insbesondere mystischen Inhalts; seit dem 16. Jh. geringere Belegdichte. - Bdv.: bekentnis 3, erkantnis, kündekeit, redlichkeit, unterscheid, unterscheidung, vernunft, vernünftigkeit, versinnigkeit, ver- 


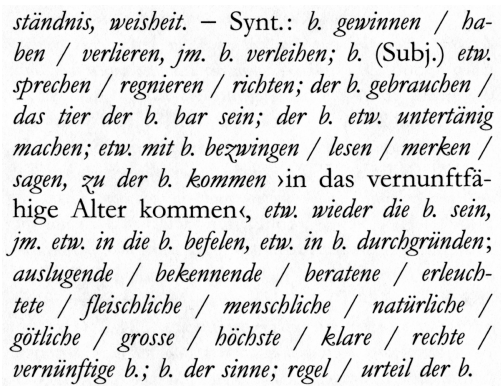

2. >Urteilsfähigkeit, Zurechnungsfähigkeit; Mündigkeit‘; vgl. bescheiden (V.) 1; 8 , bescheiden (Adj.) 2. - Wobd. / oobd.; Rechts- und Wirtschaftstexte. - Bdv.: sin, vernunft, wi - Synt.: $b$. baben, die b. kränken; bei b. sein, in der b. sein; jare / tage der $b$. >Mündigkeit, rechtsfähiges Alter`; vernünftliche b.; aufgang der $b$.

RENNEFAHRT, Stadtr. Bern 483, 48 (halem., 1456): frön Anna [..., alle die wile si in semlicher bescheidenheit ist. Molwwo, Rotes Buch Ulm 41, 11 (schwäb., 1376): da der oder die selbe person in der beschaidenhait und bn iren sinnen alz wol und alz sinneclich sien. DERTSCH, Urk. Kaufb. 1223 (schwäb., 1479): [der] auch nit zu seinen

Abb. 2: Ausschnitt aus dem Artikel bescheidenheit des FWB

Man findet dort nach der Angabe der halbfett gedruckten Lemmazeichengestalt (und den hier nicht interessierenden Angaben zur Morphologie) die erste (d.h. die vom Lexikographen unter Position 1 gestellte) Bedeutungserläuterung. Sie ist in ihrem Kernstück, der Bedeutungsangabe, ${ }^{9}$ durch einfache Anführungszeichen gekennzeichnet. Auffallend dürfte die Differenziertheit, die Verdichtung, von mir aus auch die Überladung oder Schwerverständlichkeit dieser Angabe sein; sie wird mit kritischem Auge aufgenommen werden können.

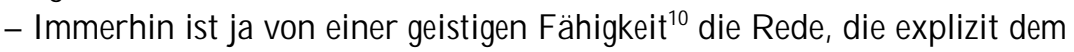
$M$ enschen, nicht etwa einem Tier zugeschrieben wird.

- Ferner wird gesagt, dass diese Fähigkeit dem Menschen von seiner natürlichen Ausstattung her zukomme oder von Gott verliehen sei; das muß den kritischen Benutzer hellhörig machen.

- Außerdem ist die Rede von einer Fähigkeit zu rationalem Utteil und zu einer implizierten $\mathrm{H}$ andlungskonsequenz dieses U rteils (nämlich der rationalen $\mathrm{K}$ ontrolle des $\mathrm{H}$ andel ns), so dass (uteil und dem U rteil entsprechendes $\mathrm{H}$ andeln - ein als Metonymie auffassbares Verhältnis - zusammenfallen.

- Es ist die Rede davon, dass dieses Uteilsvermögen in der unterscheidungsfähigkeit besteht. J eder kritische Leser wird an den Sündenfall erinnert, seit dem wir erst zwischen ,gut' und ,böse' zu unterscheiden wissen. Gleichzeitig wird damit und mit anderen Formulierungen ${ }^{11}$ der religiöse

9 Die Bedeutungsangabe bildet also nur einen Teil der viel komplexeren sog. Bedeutungserläuterung.

10 Bezugnahmen auf den Wörterbuchtext sind kursiv gesetzt.

11 Man vgl. im sog. semantischen K ommentar, der sich der Bedeutungsangabe 1 anschließt, die Formulierung: „auf religiöse Heiligung, Erkenntnis Gottes, auf Werke des Glaubens und der Liebe [...] gerichtet". 
Rahmen evoziert, innerhalb dessen eine Bedeutungseinheit wie bescheidenheit 1 steht; beim kulturhistorisch Gebildeten werden vermutlich auch das mittelalterliche Denken über Rationalität und Tugend und mit einiger Sicherheit der Unterscheidungsbegriff der Aufklärung evoziert.

- Mit den Formulierungen kluge Einsicht, Klugheit, Weisheit und Tugend wird der Bezug zur Morallehre der Zeit hergestellt.

Ich meine die Formulierungen trotz des Anspruchs, dem der Wörterbuchbenutzer mit innen unterworfen wird, verteidigen zu müssen, denn sie bringen genau das, was ich vorhin in den Punkten (2) und (3) als Forderung vorgetragen habe.

Die Differenzierung geht aber noch weiter: Ein semasiologisches Feld besteht nicht aus klar abgegrenzten, wohl unterschiedenen, sondern aus schlechtbestimmten Einheiten. Man erkennt dies sofort, wenn man sich den (ebenfalls in Abb. 2 wiedergegebenen) Bedeutungsansatz 2 zu bescheidenheit ansieht und mit dem ersten vergleicht. Ansatz 2 greift das Uteilsvermögen von Ansatz 1 in stilistischer Variation als Utteil sfähigkeit wieder auf und fügt dieser die Zurechnungsfähigkeit und (nach Semikolon) die Mündigkeit an. Klar ist also, dass es Überlappungen des einen mit dem anderen und des anderen mit den restlichen 7 , in der Abbildung aus Raumgründen nicht wiedergegebenen B edeutungsansätzen gibt. Dennoch aber liegen Gründe vor, die insgesamt 9 Ansätze einschließlich ihrer inhaltlichen Bestimmung aufrechtzuerhalten, ${ }^{12}$ auch wenn hier nicht darauf eingegangen werden kann.

W enn das FW B nun so viel mal 150.000 Erläuterungen der vorgeführten Art enthält, wie es durchschnittliche Bedeutungsansätze pro Lemmazeichen gibt, dann heißt das, dass eben genau so viele semantische Einheiten des F rühneuhochdeutschen behauptet werden, Einheiten also, in denen die Sprecher und Schreiber dieser Epoche des Deutschen ihre Darstellungs-, Kognitions- und Kommunikationstätigkeit vollzogen und in denen sie ihre Gruppenbindungen zu erkennen gegeben haben. Zu beachten ist dabei, dass die vorgenommenen Differenzierungen mit der Inhaltsnuancierung die weitere Funktion haben herauszustellen, dass auch im semasiologischen (also nicht nur im onomasiologischen) Feld kein Inhalt isoliert steht, ${ }^{13}$ sondern dass er sich aus dem Stellenwert ergibt, den das

12 Die Gründe für die F eingliederung semasiologischer F elder sind eines der Stiefkinder der Lexikographie; vgl. aber die lexikographische Einleitung des FWB, Bd. 1, S. $111 \mathrm{ff}$.

13 Hinter diesen Formulierungen stehen (wie oben schon mehrmals) die Arbeiten J . Tr ier s und in seiner Folge ein Großteil der jüngeren lexikalischen Semantik. 
gesamte Bedeutungsfeld zur Verfügung stellt. Menschliches Unterscheidungs- und uteilsvermögen ist dann eben auch immer ein solches, das in der Nähe von Zurechnungsfähigkeit und von M ündigkeit, einem rechtsrelevanten Semem, steht, sich mit diesem partiell überlappt und umgekehrt. Es gibt keine klare Trennung des einen Bereichs vom anderen. Das ist die prototypische Realisierung des vorhin genannten Punktes (3), der Lenkung der semasiologischen Aufmerksamkeit auf diejenigen Inhaltsnuancen, von denen vermutet werden kann, dass sie an einzelne Trägergruppen des Frnhd. gebunden sind.

Geht man - nach der Erwähnung von Trägergruppen - wieder zurück zur Erläuterung 1 von bescheidenheit, so findet man dort - graphisch gekennzeichnet durch den Gedankenstrich - die Angabe "gehäuft Texte religiösen, insbesondere mystischen Inhalts; seit dem 16. J ahrhundert geringere Belegdichte". Mit dieser Angabe, der prototypischen Realisierung des obigen Punktes (4), werden die Trägergruppen von Bedeutung 1 dingfest gemacht. Rhematisch ausgedrückt: Es sind diejenigen, die Texe religiösen, insbesondere mystischen Inhalts produzieren und die seit dem $16 \mathrm{~J}$ ahrhundert das W ort bescheidenheit in Bedeutung 1 nach Aussage meines Corpus mit geringerer Häufigkeit gebrauchen. Das ist keine semantische Angabe, sondern eine pragmatische, genauer: eine Symptomwertangabe, die dem zeitgenössischen Leser (in diesem Fall) textsortenbezogene und zeitbezogene (in anderen Fällen auch raumbezogene) und mit all dem sprecher- bzw. schreiberbezogene Information gibt und inn demzufolge auf einen K ommunikationsrahmen verweist, in dem der Inhalt der Bedeutungsangabe 1 gilt. Die Symptomwertangaben verdanken ihre Existenz dem Faktum, dass es das lexikalische Konstitut oder die semantische Einheit ,menschliches Unterscheidungs-, U rteilsvermögen' nicht in einem freien kognitiven Raum, sondern nur innerhalb eines bestimmten kommunikativen $\mathrm{K}$ räftefeldes gibt, in unserem Fall einem Feld, das sich in Texten religiösen Inhalts ausdrückt und im $16 \mathrm{~J}$ ahrhundert möglicherweise gewissen Veränderungen unterliegt.

Obwohl alle weiteren, oben genannten Punkte von hoher Relevanz sind und durchaus unter die kulturhistorische Perspektive gestellt werden könnten, ist hier die Setzung von Prioritäten gefordert. Ich beschränke mich auf die Angaben zur onomasiologischen Vernetzung. Man findet sie im beispielhaft ausgewählten Textausschnitt unter bescheidenheit 1 - eingeleitet durch die Sigle Bdv. (für bedeutungsverwandt) - im Anschluss an die Symptomwertangaben. Sie lauten: bekentnis 3, erkantnis, kündekeit, redlichkeit, unterscheid, unterscheidung, vernunft, vernünftigkeit, versinnigkeit, verständnis, weisheit. Das ist die Form, in der sich die Forderung (5) 
prototypisch realisiert: Kulturgeschichte ist nicht schreibbar mit dem Blick auf einzelne Sememe, und seien es quantitativ noch so viele. Da jeder Begriff (Inhalt) auf verschiedene W eise ausdrückbar ist, kann sie nur als Geschichte geschrieben werden, die von onomasiologischen Feldern (nicht von Einzelbedeutungen), in Wirklichkeit (wie wir gesehen haben) von semasiologischen und onomasiologischen Feldern in Kombination ausgeht. Zur B etonung dieses Sachverhaltes sei das Ineinandergreifen von Semasiologie und Onomasiologie in einem eigenen Schema (Abb.3) und an einem anderen B eispiel veranschaulicht.

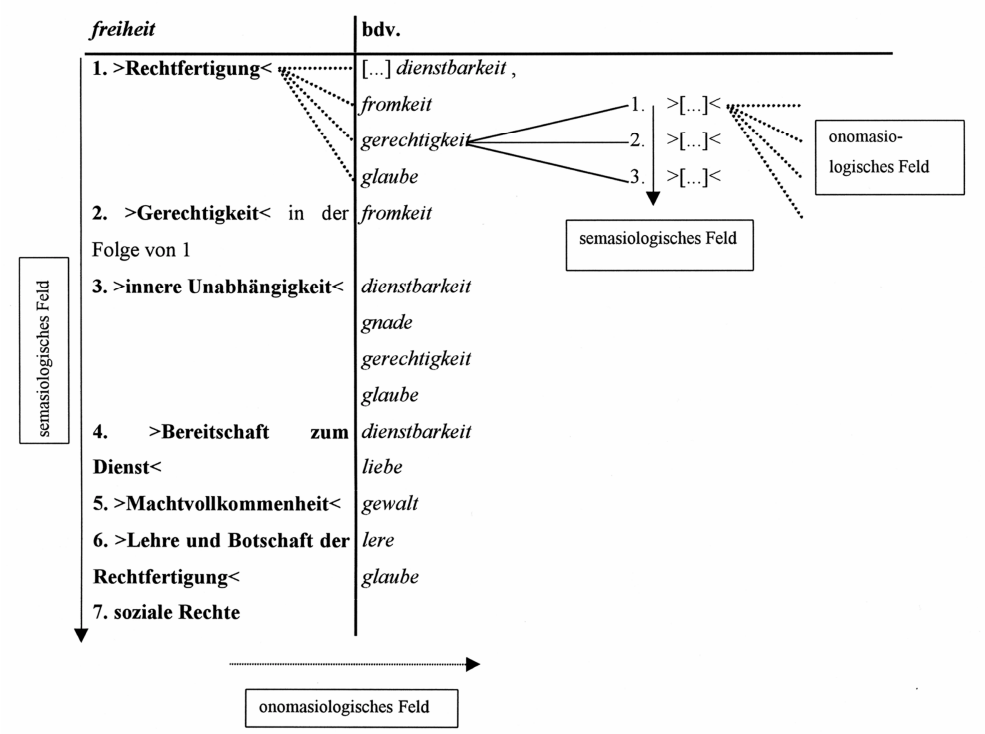

Abb. 3: Das Ineinandergreifen von Semasiologie und Onomasiologie am Beispiel frei heit

Die materiale Füllung des Schemas beruht auf den lexikalischen Ausdrücken, die Martin Luther vor allem in seiner Freiheitsschrift (1520) zur Darlegung seines Konzeptes von christlicher freiheit gebraucht hat (vgl. A. Lobenst ein-R eichmann 1998). Der zentrale Ausdruck für dieses Konzept ist freiheit, ihm können semasiologisch insgesamt 7 begründet voneinander unterschiedene Bedeutungen zugeschrieben werden. Im Schema finden sich diese (darstellungsbedingt in beschreibungssprachlicher Kurzfassung) im linken Feld entsprechend dem Senkrechtpfeil von oben nach unten unter den Zahlen 1 bis 7 abgetragen, also etwa 1. >R echtfertigung< 2. $>$ Gerechtigkeit< 4 . >Bereitschaft zum Dienst $<$ Der Inhalt jeder dieser 
Bedeutungszuschreibungen kann nun nach dem Prinzip, dass jeder Inhalt systematisch auf verschiedene Weise fassbar ist, auch mit anderen Ausdrücken gefasst werden. Für 1 . $\ngtr$ echtfertigung $<$ brauchte Luther $z$. B. die in der mittleren Spalte des Schemas stehenden, ein onomasiologisches F eld zu >Rechtfertigung< bildenden Einheiten dienstbarkeit, glaube; für 4. $>$ Bereitschaft zum Dienst< brauchte er ebenfalls dienstbarkeit, daneben liebe. Damit ist die Vernetzung aber nicht abgeschlossen, denn jede Einheit jedes der nachgewiesenen onomasiologischen Felder ist (nunmehr wieder semasiologisch betrachtet) ihrerseits mehrdeutig, was im Schema durch die leeren eckigen Klammern und die Beschriftung mittels semasiologisches Feld (mit Senkrechtpfeil) angedeutet sein soll. In dieser Weise könnte man in weiteren Betrachtungsschritten fortfahren.

Die Anwendung der kulturhistorischen Perspektive auf den Gegenstand "frühneuhochdeutscher W ortschatz" war in dem bisher Vorgetragenen bereits durchgehend impliziert. Ich will trotzdem noch einmal auf diese Beziehung eingehen. Wenn man Sprache, wie ich es nicht tue, als ein Symbolsystem fasst, mit dessen Hilfe man auf Gegenstände irgendeiner realen oder gedachten W elt Bezug nimmt, dann tendiert eine solche Auffassung dazu, die "Gegenstände" als vorsprachlich diskret (klar, deutlich unterschieden) vorauszusetzen und im Begriffssystem des Menschen (wie man gerne sagt:) abgebildet, repräsentiert zu sehen. Ihre lexikalische Fassung wäre dann lediglich als direkt sachbezügliche oder als durch die Begriffe gebrochene Fixierung von vorsprachlich oder gar vorkognitiv Vorhandenem im Zeichen zu verstehen. Die Auffassung tendiert ferner dazu, eine Proportionalität zweier linguistischer Konzeptionen zu unterstellen: Auf der einen Seite wäre das die angenommene Einheitlichkeit des Zeichens, seine Festlegung, sein Werkzeug- und Instrumentcharakter als seine sog. Äußerlichkeit (ausdrucksseitige Gestalthaftigkeit), auf der anderen Seite seine darstellungs- wie kommunikationsfunktionale Leistungsfähigkeit. J e einheitlicher, festgelegter, instrumental handhabbarer also das Zeichen, desto leistungsfähiger wären Darstellung und Kommunikation. Eine Kulturgeschichtsschreibung, die diesem Sprachverständnis huldigt, würde Sprachwissenschaft als Hilfswissenschaft verstehen, deren Aufgabe darin besteht, all die in ihrer Sicht als Verzerrung, Brechung o. ä. erscheinenden $\mathrm{K}$ onstitutionsleistungen von Sprache offenzulegen, um auf diese W eise an die Begriffe als hinter der Sprache liegende und schließlich an die Sachen als hinter den Begriffen liegende Gegebenheiten heranzukommen. Wilhelm von Humboldt kennzeichnet diese Auffassung wie folgt: "Zu verkennen“, dass "das Bezeichnete ein von seinem Zeichen unabhängiges Daseyn hat [...], ist der Grundirrtum, der alle Sprachwissenschaft 
und alle Würdigung der Sprache zerstört" (Werke 5, S. 428). Die hier vertretene Auffassung sieht sich $u$. a. in der Tradition Humboldts. Danach ist die Gesellschaft der Ort der Zeichenbildung; die Gegenstandskonstitution ist in seinem Sinne ein Gemeinsamwerden von fortwährenden Konstitutionsversuchen, sie unterliegt deshalb immer dem Geschmack, der bereits vorhandenen Kultur und der besseren Einsicht (so Coseriu 1974), auch natürlich sozialen Machtverhältnissen; sie ist ein Prozess, dessen R esultate vorübergehend einen hohen Grad an F estigkeit aufweisen mögen,

- der aber (erstens) prinzipiell Veränderung ist im Sinne der Bildung dauernd wechselnder Zeichenhalte (semantischer Aspekt),

- der (zweitens) Veränderung ist im Sinne von dauernd wechselnder Bindung von Zeichen und Zeichengebräuchen an gesellschaftliche Gruppen (pragmatischer Aspekt)

- und der (drittens) Veränderung ist im Sinne von fortwährender Konstitution gesellschaftlicher Gruppen durch Zeichengemeinsamkeiten (soziologischer Aspekt).

Sprache ist selbstverständlich auch nach dieser Auffassung immer irgendwie Mittel, das Mittel ist aber der Zeichenbildung nicht äußerlich, sondern geht konstitutiv in sie ein. Sprachwissenschaft in diesem Verständnis ist dann nicht mehr Hilfsdisziplin der Geschichtswissenschaften, sondern im wahrsten Sinne Grundlagenwissenschaft, von deren Gnaden alle von sprachlicher Überlieferung ausgehenden Wissenschaften leben. Ausgangspunkt der Betrachtung ist dann ja nicht nur die Sprache in einem methodischen Sinne (das ist unbestreitbar). Es ist vielmehr die Sprache in einem theoretischen Sinne, in vorliegendem Zusammenhang das einzelsprachliche Lexikon als seiner Seinsweise nach prozesshaftes Systemoid von Ausdrucksgestalten und nur in innen existenten Inhalten sowie von Symptomwerten aller Art, außerhalb deren kein Zugang zu einer wie auch immer vorstellbaren Wirklichkeit möglich ist, die diese Wirklichkeit (als kulturelle Wirklichkeit) großenteils ist. Als Ziel der Erkenntnis kommt dann nur der sprachgebundene, $d . h$. der einzeltextliche, textsortenspezifische, historio-, dia-, soziolektgebundene Inhalt in Betracht. Über ,Ehre' und ,Huld', ,Freiheit' und ,Glauben', über auch über ,Hagel' und ,Unwetter' zu reden, ist dann zunächst einmal (das heißt: bis zum Zugriff des Historikers, der diesen dann wissenschaftstheoretisch begründen muss) kein Reden über etwas in der Wirklichkeit oder in unseren Köpfen (in Form von Wirklichkeitsabbildern) sprachunabhängig Vorhandenes, sondern es ist ein Bezugnehmen auf etwas nur in der Sprache Vorhandenes, damit auf das Bedeutungswissen der Gesamtheit zeitgenössischer Sprecher einer Sprache sowie auf die Sicht von Realität, die sich damit verbin- 
det. Dass ein abbildtheoretisch orientierter Historiker diese Bestimmung seines Faches als Teil- und Folgedisziplin der Sprachwissenschaft nicht teilen würde, ist in Grenzen nachvollziehbar. Er müsste aber nachweisen, wie er methodisch auf etwas in sprachlich (lexikalisch, syntaktisch, textlich) verfassten Quellen Vorliegendes und nur über diese Zugängliches zu den (metaphorisch gesprochen:) dahinter liegenden Sachen oder Begriffen kommt; er müsste außerdem die theoretischen (das sind immer auch ideologischen) V orannahmen nennen, die es ihm erlauben, zu einem Verständnis von Sachen und Begriffen als letztlich vorsprachlich (sprachfrei) existierenden Gegebenheiten zu kommen.

Bezieht man das Vorgetragene auf das FWB, dann ergibt sich Folgendes:

- Indem ich die bereits mehrfach bemühten 150.000 mal x Bedeutungserläuterungen liefere,

- indem ich diese semasiologisch begründet ausdifferenziere,

- und in onomasiologische Vernetzungen stelle,

- indem ich jede der angesetzten Bedeutungen auch in ihren syntaktischen Gebräuchen und in ihrer wortbildungsmorphologischen Systematik und ihrer Nutzung in der Norm vorführe,

- indem ich außerdem ihre Trägergruppen nenne,

- und indem ich durch beschreibungssprachliche Offenheiten verschiedenster Art die Prozesshaftigkeit der Zeichenbildung und -verwendung betone,

stelle ich dem Kulturgeschichtsschreiber ebenso viele differenzierte Zeichenkonstitute (= Ausdrucks- und Inhaltskonstitute) in ihren allseitigen strukturellen (= paradigmatischen und syntagmatischen) sowie pragmatischen und soziologischen Zusammenhängen für seine Sammel-, Ordnungsund Sinngebungstätigkeit im Rahmen der von inm geleisteten Traditionssicherung und Traditionsbildung zur Verfügung. Mit diesen Konstituten (es gibt vom Zugang her keine anderen) hat er zu arbeiten und muss sich dann wissenschaftstheoretisch entscheiden, wie er die genannten sprachlichen Gegebenheiten in seine z. B. begriffs- oder sachgeschichtlichen Interessen integriert.

Diese Formulierungen schließen aus, dass Kulturgeschichte in der historischen Wortforschung im Sinne der W örter- und Sachen-F orschung auf die berühmten Sachgegenstände wie land- und hauswirtschaftliche Geräte (vom Typ ,Pflug') beschränkt wird. Eine solche Beschränkung würde schon material gesehen eine Zurückschneidung der Aufgabenstellung auf einen ihrer irrelevanteren Teilbereiche bedeuten; sie würde außerdem der hier vertretenen Sprachauffassung zuwiderlaufen. Ferner schließen die ge- 
brauchten Formulierungen die im Kern etymologiegestützten und überhaupt ausdrucksseitig orientierten $V$ erfahren zwar nicht aus, rangieren sie aber hinsichtlich ihres Gewichtes deutlich hinter den sprachinhaltsorientierten Verfahren. Der Tagungstitel „Deutsche W ortforschung als Kulturgeschichte" bringt dies übrigens treffend zum Ausdruck: Historische Wortforschung hat nicht irgendwo eine Hilfsfunktion für die Kulturgeschichtsschreibung, liefert nicht randständige $F$ älle für geschichtswissenschaftliche (dies Attribut hier im Gegensatz zu: sprach- oder bedeutungsgeschichtlich gemeint) Interessen, sondern zeichnet ein linguistisch begründetes Bild der Inhaltssysteme, in denen historische Sprechergruppen sprachlich gehandelt und gedacht haben. Insofern ist sie Kulturgeschichte im Sinne von ,Kulturgeschichtsschreibung'. F. M authner formuliert dies wie folgt: „Man wird sich daran gewöhnen müssen, in jeder Wortgeschichte eine Monographie zur Kulturgeschichte [...] zu erblicken. Sprachgeschichte, Wortgeschichte ist immer Kulturgeschichte, wenn wir den Stoff (gemeint ist der Sprachinhalt, O. R.) betrachten; eine besondere Sprachund Wortgeschichte gibt es nur für die Form" (1910/11, S. XIV). Er spricht weiter von der Geschichte "wichtiger oder wertgeschätzter Wörter", ihrer äußeren, auf die Zeichengestalt bezogenen, und ihrer inneren, auf die Bedeutung (den Sprachinhalt) bezogenen Geschichte und sieht letztere „als ein Stück geistiger Kulturgeschichte“ (ebd.).

\section{Dosierungen}

Ich habe bisher so getan, als würde das Informationsprogramm des FWB gleichsam mechanisch, Einheit für Einheit, ohne jede Gewichtung und ohne besondere Beschreibungsschwerpunkte abgefahren. Dies war gewollt, denn tatsächlich ergibt sich aus dem Vollständigkeitsideal eine Verpflichtung zur Behandlung auch des unter allen denkbaren Gesichtspunkten Uninteressanten. U mgekehrt schließst das natürlich nicht aus, dass man seine Informationen - gleichsam wie mit einem Dimmer - dosieren kann. Man kann etwa die Bedeutungserläuterung einfach und komplex, man kann sie synonymisch und phrastisch gestalten; man kann die semasiologische Differenzierung mit einem relativ hohen und einem relativ niedrigen Abstraktionsgrad realisieren, man kann die Angabe von Symptomwerten, von onomasiologischen Feldern, Syntagmen und Wortbildungen unterschiedlich stark raffen und dehnen, und man kann einen längeren oder einen kürzeren Belegteil bieten. All dies geschieht nicht willkürlich, sondern nach vorgegebenen Gesichtspunkten, die hier allerdings nicht im 
Detail erläutert werden können. Auf einige Dosierungsprinzipien soll dennoch kurz hingewiesen werden.

(1) Schon die Bedeutungsangabe ist kaum eine Definition im strengen Sinne, sondern in aller Regel eine E rläuterung, bei deren Abfassung man sich einen Benutzer vor Augen hält. Ihre Formulierung schwankt insofern zwischen den Polen ,Beschreibung' (verstanden als möglichst exakte beschreibungssprachliche Abbildung von objektartig Gedachtem, „eigentlich" Gewesenem ${ }^{14}$ im Sinne L. R ankes) und ,Behandlung' (verstanden als genuin pragmatische Vermittlung eines historischen Bezugsgegenstandes an einen heutigen Wörterbuchbenutzer). Da die Bedeutungsangabe nur das Kernstück der Bedeutungserläuterung ist, multiplizieren sich die Gestaltungsmöglichkeiten.

(2) Generell steigt der Grad der Ausführlichkeit mit dem Grad der Differenz zwischen dem historischen Bezugsgegenstand und seiner heutigen Entsprechung. Die Standardmittel, diese Differenz herauszustreichen, sind die phrastisch-komplexe Bedeutungsangabe, aber im Prinzip auch andere Informationspositionen, die die Wörterbuchanlage zur Verfügung stellt. Die von vornherein als different in Frage kommenden und der besonderen Aufmerksamkeit unterliegenden Gegenstände entstammen den sich natürlich überlappenden Bereichen ,R eligion', ,Recht', ,geistige Kultur', ,Wirtschaft', ,Herrschaft', ,Sozialität', dies alles auch im Alltagsbereich. Es geht also um die Legion von Lemmazeichen, die mit abenteuer, angst, arbeit, barmherzigkeit, gnade, grund, leben, leib nur angedeutet werden können. ${ }^{15}$ In diesem Zusammenhang darf ich daran erinnern, dass ein Teil der Corpusexzerption des FWB über Glossare zu Ausgaben frnhd. Texte lief. $D$ a diese Glossare sehr oft umfängliche semantische, darunter auch speziellste sachkundliche Erläuterungen enthalten, verfügt das FWB über große Teile der Interpretationsgeschichte des hier gemeinten religiösen, rechtlichen, sozialen, herrschafts-, wirtschafts- und allgemein kulturbezogenen Wortschatzes des Frühneuhochdeutschen. Dies bedeutet mit zunehmender Dauer der lexikographischen Tätigkeit eine zunehmende Sensibilisierung für semantische Differenzen, anders herum ausgedrückt: einen allmählichen (aber nie vollständigen) A bbau der permanenten Über-

14 Hierzu die klassische Aussage von L. R anke 1824 (in: W. Har dtwig 1990, S. 45): „Man hat der Historie das Amt, die Vergangenheit zu richten, die Mitwelt zum Nutzen zukünftiger J ahre zu belehren, beigemessen: so hoher Ämter unterwindet sich gegenwärtiger Versuch nicht: er will bloß sagen, wie es eigentlich gewesen ist."

15 Selbstverständlich könnten hier nicht nur Substantive genannt werden, sondern auch Beispiele aus allen anderen morphologischen wie funktionalen Wortarten, darunter auch z. B. die Modalpartikeln. 
forderung, der man als Lexikograph für die culture-bound words in besonderer W eise unterliegt.

(3) Die spätere frnhd. Zeit gilt als die Periode, in der sich der allmähliche Ü bergang von einer konzeptionell mündlich und pragmatisch organisierten (natürlich nicht schriftlosen), starke räumliche Fächerungen aufweisenden Zeit zu einer konzeptionell schriftlich orientierten, logik- und formbetonten Zeit vollzieht. Der gemeinte Übergang ist wissenschaftlich schwer fassbar, weil es keine klar erkennbare Bindung bestimmter lexikalischer Ausdrücke aus bestimmten Kulturbereichen an die Medien ,Schriftlichkeit' und ,M ündlichkeit' und schon gar nicht an deren Konzeption gibt. Dennoch müssen solche Bindungen vorhanden gewesen sein; ich vermute z. B., dass die gerade unter (2) angesprochenen Kulturwörter schriftlichkeitsaffiner sind als die später noch erwähnten baum und ast. Zur Gewichtung der Schriftlichkeits-Mündlichkeits-Dichotomie füge ich hinzu, dass ich persönlich dazu tendiere (es mag eine Obsession sein), für die Zeit um 1500 eine gigantische, die W ortgeographie, -soziologie und -pragmatik umfassende, sowohl soziologische, strukturelle, mediale, gebrauchs- und bewusstseins- wie kontaktgeschichtliche Umschichtung des gesamten Schreib- und Sprechhandelns, das ist meine Vertikalisierung (vgl. R eichmann 2003), anzunehmen. Wenn sie überhaupt lexikographisch fassbar sein sollte, dann über den Grad der durchschnittlichen Polysemie, den Grad der semantischen Normierung und über die Textsortenverteilung. J edenfalls erfährt der Aufmerksamkeitsdimmer bei jedem Verdacht auf eine Aussagemöglichkeit nach der Dichotomie ,Schriftlichkeit/Mündlichkeit' eine Einstellung auf volle Lichtstärke. Es geht eben darum, den ganzen Sockel von Ausdrücken zu erfassen, die der jeden einzelnen Sprecher erfassenden Literarisierung vorausgehen und nur medial schriftlich festgehalten wurden, und sie von denjenigen zu unterscheiden, die Teil eines konzeptionell schriftlichen Zugangs zur W elt sind.

(4) Die vorgetragenen 3 Punkte besagen umgekehrt, dass historisch relativ konstante Bezugsverhältnisse eine tendenziell kurze Behandlung erfahren. Dies gilt für einzelne Lemmazeichen und einzelne Bedeutungen aller Wortschatzteile. Verdächtig unter diesem Aspekt sind generell der Konkretwortschatz (mit Ausdrücken wie baum, ast) oder der Alltagswortschatz (mit dem Beispiel befürchten). Ich habe allerdings bewusst formuliert: nur „generell“" verdächtig; man tut sich nämlich schwer, überzeugende F älle zu finden, und erlebt oft Ü berraschungen (dazu der folgende Punkt 5).

(5) Auch der Konkret- und Alltagswortschatz weist für sehr viel mehr Fälle, als man gemeinhin annimmt, eine deutlich geschichtstypische Verwendung auf. Natürlich sind haber, hagel, hals, hammel und hammer, die ich 
gerade bearbeitet habe, schon immer mit Bezug auf $\mathrm{Hafer}, \mathrm{H}$ agel, Hals, Hammel und Hammer verwendet worden, insofern kann man die Bedeutungsangabe auf ein nhd. Synonym gleicher Gestalt herunterschrauben, aber ,Hagel' war in der Wahrnehmung der damaligen Zeit eine Naturgewalt, die in den U mkreis der vom Menschen verursachten Katastrophen, damit menschlicher Schuld und der Strafe Gottes gehörte; und ,Hafer' war ein Gegenstand der Versorgung der Bevölkerung mit Grundnahrungsmitteln und der Viehfütterung, der einer Fülle von Rechtsvorschriften unterlag und ganze soziale $\mathrm{H}$ andlungsfelder besetzt hielt. $\mathrm{H}$ als gehört zur Gruppe derjenigen Ausdrücke, die im Zusammenhang mit der Gesetzesübertretung eine besondere Rolle spielten und die geradezu beispielhaft für das Menschenbild frnhd. Zeit (Verbrechen, Vergänglichkeit, Strafe, Tod) stehen. Vergleichbares wäre über die Relevanz von ,Hammel' und ,H ammer' zu sagen. In all diesen, zu Beginn einer Artikelbearbeitung nicht erwartbaren Fällen ist genau darauf zu achten, wo die Kulturtypik steckt und wie sie lexikographisch vermittelt werden kann. Lexikalische Indikatoren für besondere kulturgeschichtliche E rgiebigkeit sind: häufige tropische und bildhafte Verwendungen, die Bindung an besondere, z. B. religiöse oder rechts- und wirtschaftsbezügliche Texte, eine auffallende Phrasem- und Sprichwortaffinität, ein besonders reiches onomasiologisches F eld, besonders stereotype oder variierte Syntagmen, ein vergleichsweise häufiges Eingehen in W ortbildungen. ${ }^{16} \mathrm{Für}$ all dies stellt das FWB Informationspositionen zur Verfügung, die durch Dehnung bzw. - in uninteressanten Wiederholungsfällen - durch Raffung so gestaltet werden können, dass das generelle Beschreibungsanliegen des FWB, die Dokumentation des spätmittelalterlichen und früneuzeitlichen Wortschatzes und des kulturtypischen Bedeutungswissens seiner Träger, herausprofiliert wird.

(6) Zum Schluss dieses Abschnitts weise ich noch darauf hin, dass die vorhin vorgestellte Artikelstruktur des FWB für jede Informationsposition die Möglichkeit eines Kommentars vorsieht. Es gibt also z. B. einen Kommentar zum Lemmaansatz, zur Bedeutungserläuterung, zu den Symptomwerten, zur Angabe der onomasiologischen Vernetzung, zu den Syntagmen usw. bis hin zu den Belegen. Aufgabe dieser natürlich sparsam gehandhabten und mit den beschreibenden Angaben verwobenen Kommentare ist die Mitteilung von Überlegungen, Vermutungen, Hypothesen, Zweifeln, aber auch die Anbringung inhaltsstrukturierender Verweise. Sie sind damit ein weiterer Ort für Dosierungen.

\footnotetext{
${ }^{16}$ Dies alles ist nachschlagbar in Lieferung 2 von Band 7, die im Herbst 2004 erscheint.
} 


\section{Schl uss}

Vorliegender Artikel hat eine methodische, vor allem aber eine theoretische, speziell geschichtstheoretische Ausrichtung. Es wird also beschrieben, wie der Wortschatz eines bestimmten Sprachstadiums, hier des F rühneuhochdeutschen, verstanden und lexikographisch behandelt werden muss, wenn er Gegenstand von Kulturgeschichtsschreibung sein soll. Die gleichen oder mindestens analoge Aussagen hätte man zum Alt-, Mitteloder Neuhochdeutschen machen können. Es wird also (umgekehrt ausgedrückt) nicht beschrieben, welche besonderen Kennzeichen der objektsprachlich vorhandene W ortschatz des F rühneuhochdeutschen unter kulturgeschichtlicher Perspektive etwa im Unterschied zu demjenigen dieser anderen Sprachstadien des Deutschen aufweist. Da dies aber ein sprachgeschichtlich kaum untersuchtes und hoch relevantes Thema ist, seien eingeschränkt auf den Vergleich mit dem Neuhochdeutschen - wenigstens einige, teilweise provokante objektsprachliche Charakterisierungen vorgetragen:

(1) Der frnhd. Wortschatz ist, wie selbst durch den Filter der Schriftlichkeit erkennbar ist, umfangreicher als der neuhochdeutsche.

(2) Er ist deutlich stärker varietätengebunden als der neuhochdeutsche.

(3) Er zeigt möglicherweise einen höheren Polysemiegrad als der Wortschatz des gesamten Neuhochdeutschen (Hochsprache, Raum-, Sozialund F unktionalvarietäten).

(4) Er spiegelt den Konflikt zwischen den genannten beiden Seinsweisen von Sprache, der konzeptionell mündlichen und der schriftlichen, anders als der neuhochdeutsche.

(5) Er zeigt generell eine viel stärkere religiöse Durchdringung als der neuhochdeutsche, überhaupt bereichsspezifisch andere Ausbauformen.

(6) Er zeigt eine im Vergleich zur neuhochdeutschen Hochsprache besondere onomasiologische Vielfalt im N egativbereich.

(7) Er zeigt eine vergleichsweise hohe Phrasemaffinität.

Mir ist bewusst, dass jeder dieser Punkte erläuterungs- und diskussionsbedürftig ist und möglicherweise nicht verteidigt werden kann, ich bin aber der Meinung, dass ein Sprechen über die Objektgegebenheit, frnhd. W ortschatz' an diesen Punkten ansetzen müsste. Dabei würde folgende Differenz deutlich werden: einerseits eine Sprachstufe (Frühneuhochdeutsch), die durch ausgeprägte geographische $K$ ämmerungen, durch ein besonderes Verhältnis von Schriftlichkeit und Mündlichkeit, durch eine eher hori- 
zontale Lagerung ihrer Varietäten und dem allem entsprechende sprachsystematische Eigenheiten gekennzeichnet ist, und andererseits eine Sprachstufe (Neuhochdeutsch), die durch eine alles überdachende Leitvarietät literaler Provenienz, eine Verweisung großer Teile des Varietätenspektrums auf einen Platz im Substandardbereich und durch eine dem allem entsprechende Systematizität gekennzeichnet ist. Der Aufweis dieser Differenz wäre wieder Kulturgeschichte.

\section{Liter at ur (in Auswahl)}

Bahner, Werner/Neumann, Werner (Hrsg., 1985).: Sprachwissenschaftliche Germanistik. Ihre Herausbildung und Begründung. Berlin.

Barthes, Roland (1957; 2003): Mythen des Alltags. Aus dem Französischen von Helmut Scheffel. Frankfurt a. M. Sonderausgabe 2003.

Coser iu, Eugenio (1974): Synchronie, Diachronie und Geschichte. Das Problem des Sprachwandels. Übersetzt von Helga Sohre. München (Internationale Bibliothek für Allgemeine Linguistik 3).

[FWB =] Frühneuhochdeutsches Wörterbuch. Hrsg. von R. R. Ander son [für Bd. 1], Ulrich Goebel, Anja L obenst ein-R eichmann [für Bd. 6; 11-13], Oskar R eichmann. Bearb. von Oskar R eichmann [Bd. 1; 2; 4], J oachim Schil dt [Bd. 3; 6, Lief. 1], Vibeke W inge [Bd. 8, Lief. 1 und 2], Anja Lobenst ein-R eichmann [Bd. 7, Lief. 1; 9, Lief. 1 und 2]. Berlin, New York $1989 \mathrm{ff}$.

H umbol dt, Wilhelm von (1903-1936): Werke. Hrsg. von Albert Leitzmann. 17 Bde. Berlin (Wilhelm von Humboldts Gesammelte Schriften. Erste Abt.).

Lobenst ein-R eichmann, Anja (1998): F reiheit bei Martin Luther. Lexikographische Textanalyse als Methode historischer Semantik. Berlin, New York (Studia Linguistica Germanica 46).

Mauthner , Fritz (1910/11): Wörterbuch der Philosophie. Neue Beiträge zu einer K ritik der Sprache. 2 Bde. Zürich 1980. Nachdruck der Erstausgabe von 1910/11.

Ranke, Leopold (1824/1990): Vorrede zu den "Geschichten der romanischen und germanischen Völker von 1494 bis 1535“. In: Über das Studium der Geschichte. Hrsg. v. Wolfgang $H$ ar dt wig (DTV 4546). München 1990, S. 42-47.

R eichmann, Oskar (1986): Lexikographische Einleitung. In: FWB, Bd. 1, Lief. 1, S. 10-164.

R eichmann, Oskar (1990a): Das gesamtsystembezogene Wörterbuch. In: W örterbücher, Teilbd. 2, S. 1391-1416.

R eichmann, Oskar (1990b): Formen und Probleme der Datenerhebung I: Synchronische und diachronische historische W örterbücher. In: W örterbücher, Teilbd. 2, S. 1588-1611.

R eichmann, Oskar (2002a): Vorwort. In: FWB, Bd. 3, S. V-XXV.

R eichmann, Oskar (2002b): Wortbildungsfelder des Frühneuhochdeutschen. In: Historische Wortbildung des Deutschen. Aufbau, Probleme ihrer lexikographischen Behandlung, sprachgeschichtliche Perspektiven. Hrsg. von Mechthild Haber mann, Peter O. M ül I er , Horst Haider Munske Tübingen, S. 245-267 (R eihe Germanistische Linguistik 232).

R eich mann, Oskar (2003): Die Entstehung der neuhochdeutschen Schriftsprache. Wo bleiben die Regionen? In: Die deutsche Schriftsprache und die Regionen. Entstehungsgeschicht- 
liche F ragen in neuer Sicht. Hrsg. von Raphael B er thel e, Helen Chr ist en, Sibylle Ger man, Ingrid H ove. Berlin, New Y ork (Studia Linguistica Germanica 65), S. 29-57.

Trier , J ost (1973): Aufsätze und Vorträge zur Wortfeldtheorie. Hrsg. von Anthony van der Lee, Oskar R eich mann (I anua Linguarum, Series Minor 174). The Hague, Paris.

V ier haus, Rudolf (1974): Ranke und die Anfänge der deutschen Geschichtswissenschaft. In: Geschichtswissenschaft in Deutschland. Traditionelle Positionen und gegenwärtige Aufgaben. Mit Beiträgen v. Andreas D or pal en [u. a.]. Hrsg. v. Bernd F aul enbach. München (Beck'sche Schwarze Reihe 111), S. 17-34.

Wörterbücher. Ein internationales Handbuch zur Lexikographie. Hrsg. von Franz J osef H ausmann, Oskar Reichmann, Herbert Ernst W iegand, Ladislav Zgusta (1998; 1990; 1991): 3 Teilbde. Berlin, New York (Handbücher zur Sprach- und Kommunikationswissenschaft 5).. 